Case Report

\title{
Ponatinib as a Valid Alternative Strategy in Patients with Blast Crisis-Chronic Myeloid Leukemia Not Eligible for Allogeneic Stem Cells Transplantation and/or Conventional Chemotherapy: Report of a Case
}

\author{
Cristina Bucelli, ${ }^{1}$ Daniele Cattaneo, ${ }^{1}$ Valeria Ferla, ${ }^{1}$ Manuela Zappa, \\ Caterina de Benedittis, ${ }^{2}$ Simona Soverini, ${ }^{2}$ and Alessandra Iurlo ${ }^{1}$ \\ ${ }^{1}$ Hematology Division, IRCCS Ca' Granda-Maggiore Policlinico Hospital Foundation and University of Milan, Milan, Italy \\ ${ }^{2}$ Department of Experimental, Diagnostic and Specialty Medicine, Institute of Hematology "L. and A. Seragnoli," \\ University of Bologna, Bologna, Italy \\ Correspondence should be addressed to Alessandra Iurlo; aiurlo@policlinico.mi.it
}

Received 11 April 2017; Revised 19 June 2017; Accepted 12 July 2017; Published 14 August 2017

Academic Editor: Stephen Langabeer

Copyright (c) 2017 Cristina Bucelli et al. This is an open access article distributed under the Creative Commons Attribution License, which permits unrestricted use, distribution, and reproduction in any medium, provided the original work is properly cited.

\begin{abstract}
Currently, imatinib and dasatinib are the only tyrosine-kinase inhibitors approved in the US and Europe for the treatment of blast crisis of chronic myeloid leukemia (BC-CML) at diagnosis, while ponatinib is the only inhibitor used in patients bearing T315I mutation. Here we report the case of a 61-year-old man diagnosed with B-cell lymphoid BC-CML, initially treated with imatinib $800 \mathrm{mg}$ day and then with dasatinib $140 \mathrm{mg}$ day because of intolerance. A complete cytogenetic response (CCyR) was achieved at three months; however, three months later a relapse was observed, and the T315I mutation was detected. Ponatinib $45 \mathrm{mg}$ once daily was then started together with a short course of chemotherapy. Bone marrow evaluation after six months of therapy showed the regaining of CCyR, together with the achievement of a deep molecular response. However, one year from ponatinib start the patient experienced a new disease relapse; he was effectively treated with ponatinib and chemotherapy once again, but in the meanwhile an ischemic stroke was detected. This case report confirms the high efficacy of ponatinib monotherapy in BC-CML patients, representing a valid option for non-allogeneic stem cells transplantation eligible cases and the only one available for those carrying the T315I mutation.
\end{abstract}

\section{Introduction}

Chronic myeloid leukemia (CML) prognosis changed dramatically with the development of tyrosine-kinase inhibitors (TKIs) that potently interfere with the interaction between the $B C R-A B L 1$ protein and adenosine triphosphate (ATP), thus blocking proliferation of the malignant cellular clone [1]. This targeted approach has markedly increased survival of CML patients and greatly reduced the frequency of blast crisis (BC) compared to the pre-TKI era. In fact, the incidence of $\mathrm{BC}$ now ranges between 0.7 and $4.5 \%$, showing the highest incidence in the first year after diagnosis and then decreasing $[2,3]$.

A percentage of patients between 0.9 and $6.7 \%$ are already in BC at diagnosis [2-5]. More recently, in the EUTOS population-based registry, the incidence of $\mathrm{BC}$ at diagnosis was found to be about 2.2\% [3]. Median survival ranges between 6 and 11.8 months [6].

According to the European LeukemiaNet (ELN) recommendations, $\mathrm{BC}$ is defined using the threshold blast count in the peripheral blood or bone marrow at 30\% [7].

Up to $80 \%$ of all BC patients show additional chromosomal aberrations (ACA) [8], and at least $77 \%$ carry mutations in the $A B L 1$ domain. Therefore, their mutational status should also be evaluated, since it is associated with different prognoses, for example, T315I mutation-positive cases compared to others [9-11].

At diagnosis, treatment mainly depends on the type of $\mathrm{BC}$ (myeloid versus lymphoid), mutational status, and previous therapies. Treatment options may include TKIs, conventional 


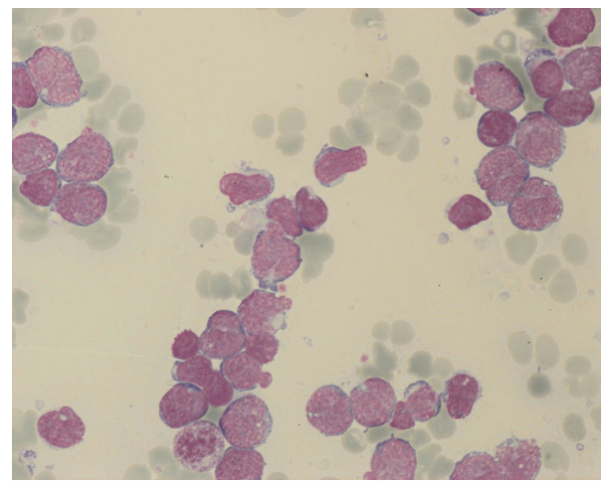

FIGURE 1: Bone marrow aspirate morphology at diagnosis, showing atypical granular lymphoblasts.

chemotherapy, allogeneic stem cells transplantation (HSCT), and/or other treatment modalities. To date, imatinib and dasatinib are the only TKIs approved in the US and Europe for treatment of $\mathrm{BC}$ at diagnosis, while ponatinib is the only one which can be used also in patients harboring T315I mutation [12-16].

Here we report the case of a 61-year-old man diagnosed with B-cell lymphoid BC-CML initially treated with imatinib, which was soon suspended because of intolerance, followed by dasatinib. Then, he acquired a T315I mutation but achieved a deep molecular response (MR4) on ponatinib therapy. Furthermore, ponatinib monotherapy was able to maintain this patient in CML-chronic phase for about one year before he died because of an infective complication.

\section{Case Presentation}

In January 2015, a 61-year-old man was referred to our hospital because of asthenia, diffuse bone pain, fever, and cutaneous hemorrhagic diathesis. Laboratory tests showed a mild anemia (hemoglobin, $12 \mathrm{~g} / \mathrm{dL}$ ), leukocytosis (white blood cells count, $22 \times 10^{9} / \mathrm{L}$, with neutrophils $57 \%$; lymphocytes $11 \%$; monocytes $2 \%$; eosinophils $6 \%$; basophils $1 \%$; myelocytes $6 \%$; metamyelocytes $4 \%$; blast cells $13 \%$ ), and severe thrombocytopenia (platelet count, $4 \times 10^{9} / \mathrm{L}$ ). The patient was obese and suffered from chronic bronchitis; in addition, he was an active smoker (about 40 cigarettes per day) and an alcohol abuser, with three previous episodes of suicide attempt. Bone marrow morphological analysis showed an atypical granular lymphoblastic infiltration of $70 \%$ (Figure 1), which was confirmed by flow cytometry immunophenotyping (high positivity for CD10/CD19/CD34/CD99/CD79a/TdT; weak positivity for CD33 (60\%)/CD38 (80\%); negativity for CD3/CD13/CD14/CD20/CD117/MPO). Cytogenetic study of 20 metaphases revealed a typical 46, XY, t $(9 ; 22)(\mathrm{q} 34 ; \mathrm{q} 11.2)$ translocation, and qualitative polymerase chain reaction (PCR) demonstrated the presence of the $B C R-A B L 1$ fusion transcript, with a typical e13a2 configuration. Baseline assessment of $B C R-A B L 1$ mutational status with Sanger sequencing was negative, datum which was also retrospectively confirmed by ultradeep sequencing. Therefore, a diagnosis of Bcell lymphoid BC of CML was made. As a matched sibling donor was not available and the patient categorically refused HSCT, we decided not to start a search for a matched unrelated donor. Because of a previous exposition to hepatitis $\mathrm{B}$ virus, a prophylactic treatment with lamivudine was initiated.

The patient was initially treated with imatinib $800 \mathrm{mg}$ day, which was suspended approximately after one month because of intolerance (grade III crippling cramps, periorbital edema, and conjunctival hemorrhage), and then he started dasatinib $140 \mathrm{mg}$ day. At the same time, methylprednisolone was administered at a full dose and then decreased for the appearance of diabetes which required treatment with oral hypoglycemic drugs and then also with insulin.

Hematological response was obtained after one month, and at three months bone marrow evaluation showed a complete cytogenetic response (CCyR), together with a $B C R$ $A B L 1$ transcript level of $0.2 \%$ according to the International Scale (IS).

Nevertheless, three months later a BC-CML relapse was observed, with a $B C R-A B L 1$ transcript level of $89.5 \%$ according to IS, and a new evaluation of $B C R-A B L 1$ mutational status revealed the presence of T315I mutation. Cytological and flow cytometric analyses of cerebrospinal fluid showed no leukemic cells.

As suggested by ELN 2013 recommendations, ponatinib $45 \mathrm{mg}$ once daily was started together with chemotherapy (vincristine intravenously every seven days for three cycles and oral prednisone). Due to patient's comorbidities, an antiplatelet therapy with ASA $100 \mathrm{mg}$ day was coprescribed in order to reduce the risk of arterial thrombotic events.

After three months of ponatinib therapy, bone marrow evaluation showed the regaining of a CCyR and a $B C R-A B L 1$ transcript of less than $10 \%$ according to IS.

The drug was fairly well tolerated, even though after two months severe upper abdominal burning pain irradiating to the back appeared. Blood analysis showed an increase in lipase and amylase levels (grade III pancreatic toxicity), and an abdomen CT scan showed a mild pancreatitis. It was then decided to maintain ponatinib at the same dosage and to strictly monitor the patient, achieving then a spontaneous resolution of all symptoms.

Bone marrow evaluation after six months of therapy confirmed CCyR, together with the achievement of a deep 


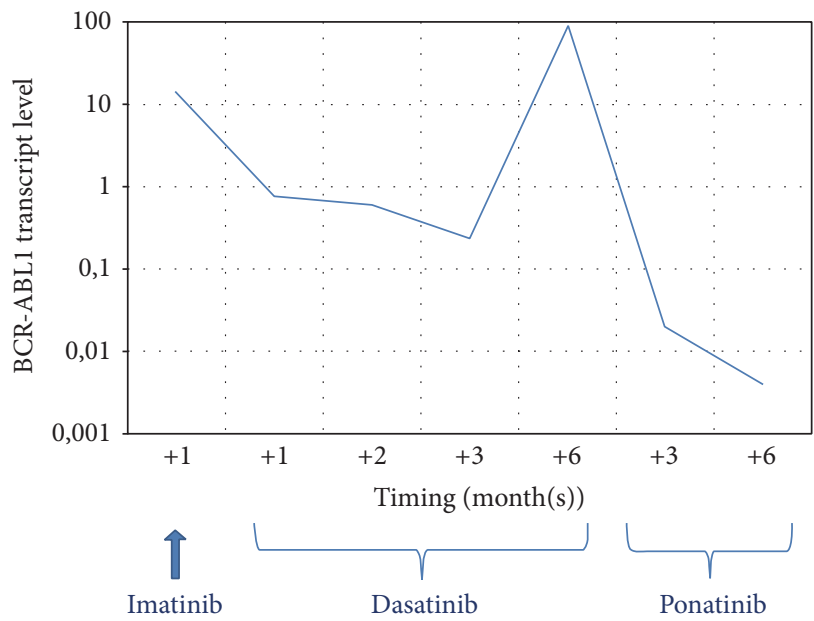

FIgURE 2: $B C R-A B L 1$ transcript level during TKIs therapy.

molecular response, with a $B C R-A B L 1$ transcript level of $0.004 \%$ according to IS (MR4) (Figure 2).

Nevertheless, after about one year from ponatinib start, the patient experienced disease relapse: bone marrow morphological analysis showed an atypical granular lymphoblastic infiltration of 55\%, which was confirmed by flow cytometry immunophenotyping (high positivity for CD10/CD19/ CD79a/CD99/TdT; weak positivity for CD33 (30\%)/CD38 (50\%); negativity for CD20/CD34). In addition, a new cytogenetic abnormality was detected, his karyotype being the following: 46,XY,t (9;22)(q34,q11.2) [3]/47,XY,t(9;22)(q34;q11.2), $+\operatorname{der}(1 ; 19)(\mathrm{p} 11 ; \mathrm{q} 11)[4]$.

Due to the good response to the previous chemotherapy, a new course of intravenous vincristine and oral prednisone was started. However, after the second cycle of chemotherapy, the patient showed dysarthria and weakness of the right leg; therefore he was hospitalized to clarify the origin of these new neurological symptoms. With the hypothesis of a central nervous system (CNS) localisation of lymphoid BC-CML, a new cytological and flow cytometric analysis of cerebrospinal fluid was performed but it showed no leukemic cells; on the contrary, a CNS CT scan demonstrated an ischemic stroke with numerous recent lesions in the periventricular area and within the frontal-parietal cortex of the left hemisphere. Accordingly, due to the vascular etiology of the ischemic event, anticoagulant therapy with low molecular weight heparin (LMWH) was added to ASA, and statins and antihypertensive therapies were started. Meanwhile, ponatinib and intravenous vincristine were regularly administered, leading to the rapid achievement of a complete normalisation of blood cells count with no need of red blood cells or platelets transfusion.

Approximately after two months from hospitalization, the patient developed a septic shock due to a pulmonary infection not responsive to empiric antibiotic therapy and died.

\section{Discussion}

Even if TKIs have completely changed the outcome and survival of CML patients, treatment of advanced phases still remains a clinical challenge, particularly for patients not eligible to HSCT, which remains to date the only potentially curative strategy for eligible patients.

In the case reported here, the patient was not eligible to HSCT due to his comorbidities correlated with a Sorror score of 8 , identifying a 2 -year non-relapse mortality of $41 \%$ and a 2 -year overall survival of $34 \%$ [17].

In addition, due to the complexity of psychological characteristics of the patient who refused not only HSCT but also hospitalization, he was initially treated with TKI monotherapy. As reported in the literature, hematologic and cytogenetic responses with single agent TKIs are achieved in about 50 and $12 \%$ of the cases, respectively, with the 12 -month overall response ranging between 25 and $49 \%$ and the median survival between 6 and 11.8 months [6]. Outcome of lymphoid BC-CML treated with dasatinib varies between 39 and $80 \%$ of hematologic remission and from 40 to $90 \%$ of any cytogenetic response $[6,18,19]$. Concerning molecular response, no data have been reported in the literature so far. Of note, in a retrospective analysis aimed at characterizing mutation development in patients with newly diagnosed CML treated with imatinib or dasatinib frontline, a narrower spectrum of mutations in dasatinib-treated patients was found, with the occurrence of T315I mutation in approximately $17 \%$ of cases [20]. Indeed, our patient experienced disease relapse within six months of dasatinib treatment with the occurrence of T315I mutation.

Since the patient was in $\mathrm{BC}$ at the time of diagnosis, he was investigated for $B C R-A B L 1$ mutational status by Sanger sequencing and found negative. However, Soverini et al. [21] and Baer et al. [22] reported that the T315I mutation can be identified by means of ultradeep sequencing approximately three months before the Sanger sequencing detection threshold is reached. Therefore, the same specimen was retrospectively analyzed by ultradeep sequencing, but despite a sequencing depth of 7614 reads (theoretically enabling a sensitivity of approximately $0.03 \%$ ) no T315I mutation was detected. Interestingly, in $4 \%$ of all alleles, a 35 bp insertion, already reported in the literature as "35INS," was identified. 
The contribution of this insertion to TKI resistance is still a matter of debate. However, a recent functional in vitro study has indicated that the $B C R-A B L 1^{35 I N S}$ isoform is not a functional kinase, thus it should not have any role in sustaining the disease and triggering drug resistance [23].

Due to the low incidence of BC-CML, studies on the combination of TKIs with chemotherapy are rather scanty and usually with a limited number of enrolled patients. Considering that patients with lymphoid BC can be treated with acute lymphoblastic leukemia- (ALL-) type induction regimens, with our patient being not eligible to HSCT, we decided to administer three courses of vincristine and high dose corticosteroids in association with ponatinib. Since only ponatinib shows activity also against T315I mutation, it was administered at full dose in our patient, despite its potential side effect profile. As reported in phase 2 PACE study of ponatinib in $\mathrm{Ph}+$ leukemias, the median time to a major hematologic response in BC-CML patients was 4.1 weeks (range, 1.7 to 16.1), and the estimated rate of a sustained response at 12 months was $42 \%$. Overall survival was estimated to be $29 \%$ at 12 months (median, 7 months), but no data were reported about achievement of major molecular response (MMR) [16].

Response to treatment is the most important prognostic factor for survival in BC-CML patients $[24,25]$. In particular, best results are obtained in patients returning to chronic phase especially if cytogenetic or molecular responses are achieved.

Concerning ponatinib safety profile, arterial thrombotic events are reported in about $22 \%$ of all treated patients, showing a linear correlation with the drug dosage [16]. It has to be considered that our patient bears different cardiovascular risk factors, including hypertension, hypercholesterolemia, steroid-induced diabetes, alcohol abuse, smoking habit, and obesity; however, ponatinib at a dosage of $45 \mathrm{mg}$ day represented the only possible therapeutic option for such a complex subject.

Our data confirm the high efficacy of ponatinib in BCCML patients and the potent activity of this drug also in achieving molecular response in such patients and maintaining the chronic phase of the disease for almost one year. In addition, another significant aspect in this case is represented by the good quality of life for the patient, who remained an outpatient for the entire treatment duration.

In conclusion, ponatinib monotherapy or in association with chemotherapy is a valid treatment option for non-HSCT eligible BC-CML patients and the only one available for those carrying the T315I mutation, which can develop during dasatinib therapy frequently.

\section{Disclosure}

This study was not conducted with research intervention thus ethics committee approval was not necessary. No funding has been available other than that of the author's institution.

\section{Conflicts of Interest}

The authors declare they have no conflicts of interest.

\section{References}

[1] H. Kantarjian, S. O’Brien, E. Jabbour et al., "Improved survival in chronic myeloid leukemia since the introduction of imatinib therapy: a single-institution historical experience," Blood, vol. 119, no. 9, pp. 1981-1987, 2012.

[2] S. Saußele, L. Kalmanti, M. Lauseker et al., "Management and outcome of CML-blast crisis: results from the randomized CML study IV," Haematologica, p. P275, 2014.

[3] D. Lindoerfer, V. S. Hoffmann, G. Rosti et al., "The EUTOS population-based registry: evaluation of baseline characteristics and first treatment choices of 3045 newly diagnosed chronic myeloid leukemia (CML) patients from 20 European countries," Haematologica, p. S680, 2014.

[4] E. J. Jabbour, T. P. Hughes, J. E. Cortés, H. M. Kantarjian, and A. Hochhaus, "Potential mechanisms of disease progression and management of advanced-phase chronic myeloid leukemia," Leukemia and Lymphoma, vol. 55, no. 7, pp. 1451-1462, 2014.

[5] R. Hehlmann, "How I treat CML blast crisis," Blood, vol. 120, no. 4, pp. 737-747, 2012.

[6] R. Hehlmann and S. Saussele, "Treatment of chronic myeloid leukemia in blast crisis," Haematologica, vol. 93, no. 12, pp. 17651769, 2008.

[7] M. Baccarani, G. Gugliotta, F. Castagnetti, S. Soverini, and G. Rosti, "European LeukemiaNet recommendations for the management of chronic myeloid leukemia," Blood, vol. 122, pp. 872-884, 2013.

[8] U. Bacher, T. Haferlach, W. Hiddemann, S. Schnittger, W. Kern, and C. Schoch, "Additional clonal abnormalities in Philadelphia-positive ALL and CML demonstrate a different cytogenetic pattern at diagnosis and follow different pathways at progression," Cancer Genetics and Cytogenetics, vol. 157, no. 1, pp. 53-61, 2005.

[9] F. E. Nicolini, A. R. Ibrahim, S. Soverini et al., "The BCRABLT315I mutation compromises survival in chronic phase chronic myelogenous leukemia patients resistant to tyrosine kinase inhibitors, in a matched pair analysis," Haematologica, vol. 98, no. 10, pp. 1510-1516, 2013.

[10] V. Grossmann, A. Kohlmann, M. Zenger et al., "A deep-sequencing study of chronic myeloid leukemia patients in blast crisis (BC-CML) detects mutations in $76.9 \%$ of cases," Leukemia, vol. 25 , no. 3, pp. 557-560, 2011.

[11] S. Soverini, A. Hochhaus, F. E. Nicolini et al., "BCR-ABL kinase domain mutation analysis in chronic myeloid leukemia patients treated with tyrosine kinase inhibitors: recommendations from an expert panel on behalf of european leukemianet," Blood, vol. 118, no. 5, pp. 1208-1215, 2011.

[12] A. M. Lewis, W. D. Croughan, N. Aranibar et al., "Understanding and controlling sialylation in a CHO Fc-fusion process," PLOS ONE, vol. 11, no. 6, p. e0157111, 2016.

[13] Gleevec, Novartis Pharmaceuticals Corporation, East Hanover, NJ, USA, 2012.

[14] Tasigna, Novartis Pharmaceuticals Corporation, East Hanover, NJ, USA, 2012.

[15] Bosulif, Pfizer Labs, New York, NY, USA, 2012.

[16] J. E. Cortes, H. Kantariian, N. P. Shah et al., "Ponatinib in refractory philadelphia chromosome-positive leukemias," New England Journal of Medicine, vol. 367, no. 22, pp. 2075-2088, 2012.

[17] M. L. Sorror, M. B. Maris, R. Storb et al., "Hematopoietic cell transplantation (HCT)-specific comorbidity index: a new tool 
for risk assessment before allogeneic HCT," Blood, vol. 106, no. 8, pp. 2912-2919, 2005.

[18] M. Talpaz, N. P. Shah, H. Kantarjian et al., "Dasatinib in imatinib-resistant Philadelphia chromosome-positive leukemias," New England Journal of Medicine, vol. 354, no. 24, pp. 2531-2541, 2006.

[19] G. Saglio, A. Hochhaus, Y. T. Goh et al., "Dasatinib in imatinibresistant or imatinib-intolerant chronic myeloid leukemia in blast phase after 2 years of follow-up in a phase 3 study: efficacy and tolerability of 140 milligrams once daily and 70 milligrams twice daily," Cancer, vol. 116, no. 16, pp. 3852-3861, 2010.

[20] T. P. Hughes, G. Saglio, A. Quintás-Cardama et al., "BCRABL1 mutation development during first-line treatment with dasatinib or imatinib for chronic myeloid leukemia in chronic phase," Leukemia, vol. 29, no. 9, pp. 1832-1838, 2015.

[21] S. Soverini, C. De Benedittis, K. M. Polakova et al., "Nextgeneration sequencing for sensitive detection of BCR-ABL1 mutations relevant to tyrosine kinase inhibitor choice in imatinib-resistant patients," Oncotarget, vol. 7, no. 16, pp. $21982-$ 21990, 2016.

[22] C. Baer, W. Kern, S. Koch et al., "Ultra-deep sequencing leads to earlier and more sensitive detection of the tyrosine kinase inhibitor resistance mutation T315I in chronic myeloid leukemia," Haematologica, vol. 101, no. 7, pp. 830-838, 2016.

[23] T. O'Hare, M. S. Zabriskie, C. A. Eide et al., “The BCRABL35INS insertion/truncation mutant is kinase-inactive and does not contribute to tyrosine kinase inhibitor resistance in chronic myeloid leukemia," Blood, vol. 118, no. 19, pp. 52505254, 2011.

[24] F. Cervantes, M. Rozman, J. Rosell, A. Urbano-Ispizua, E. Montserrat, and C. Rozman, "A study of prognostic factors in blast crisis of Philadelphia chromosome-positive chronic myelogenous leukaemia," British Journal of Haematology, vol. 76, no. 1, pp. 27-32, 1990.

[25] J. Wadhwa, R. M. Szydlo, J. F. Apperley et al., "Factors affecting duration of survival after onset of blastic transformation of chronic myeloid leukemia," Blood, vol. 99, no. 7, pp. 2304-2309, 2002. 


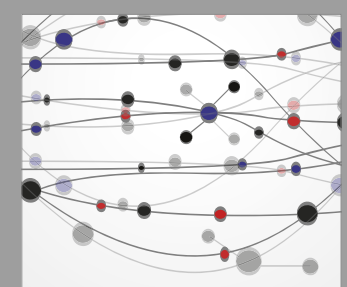

The Scientific World Journal
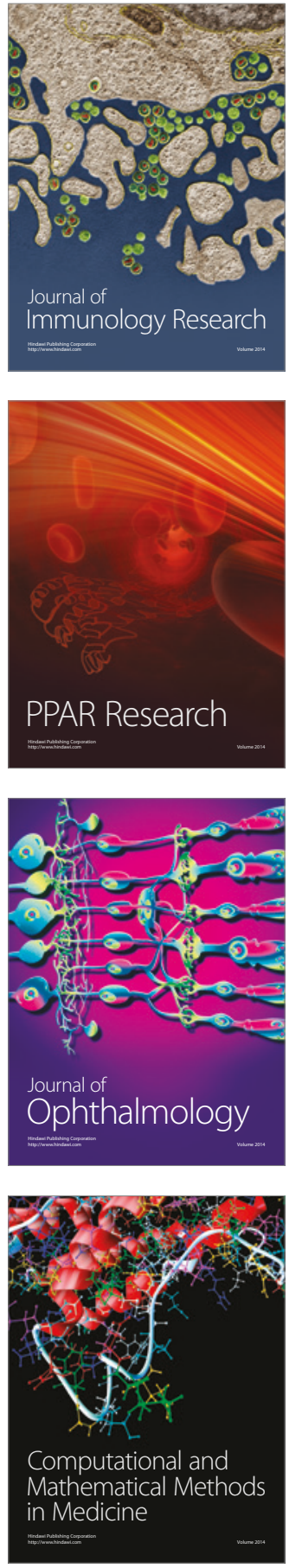

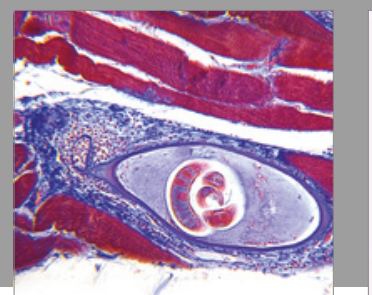

Gastroenterology Research and Practice
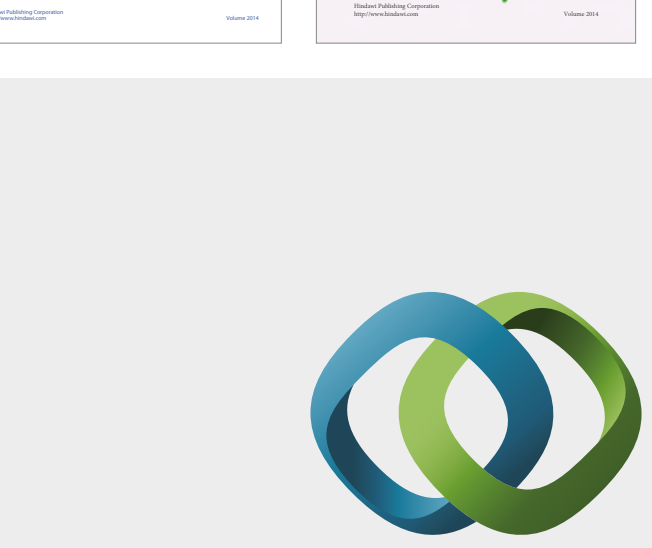

\section{Hindawi}

Submit your manuscripts at

https://www.hindawi.com
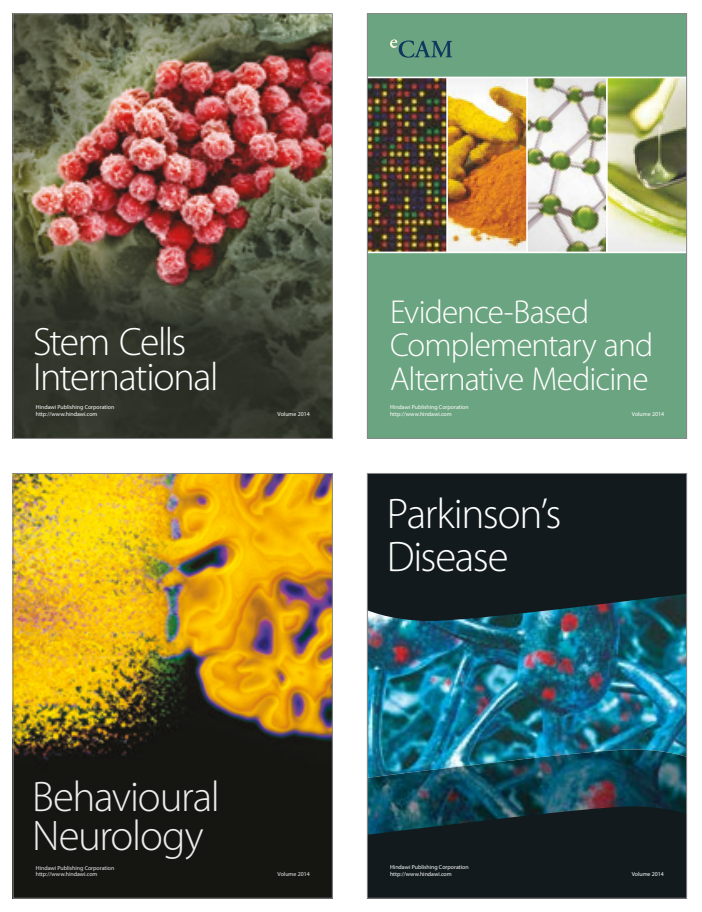
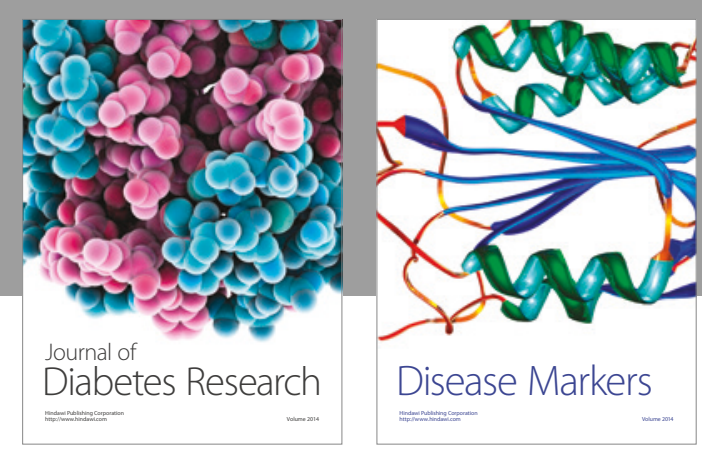

Disease Markers
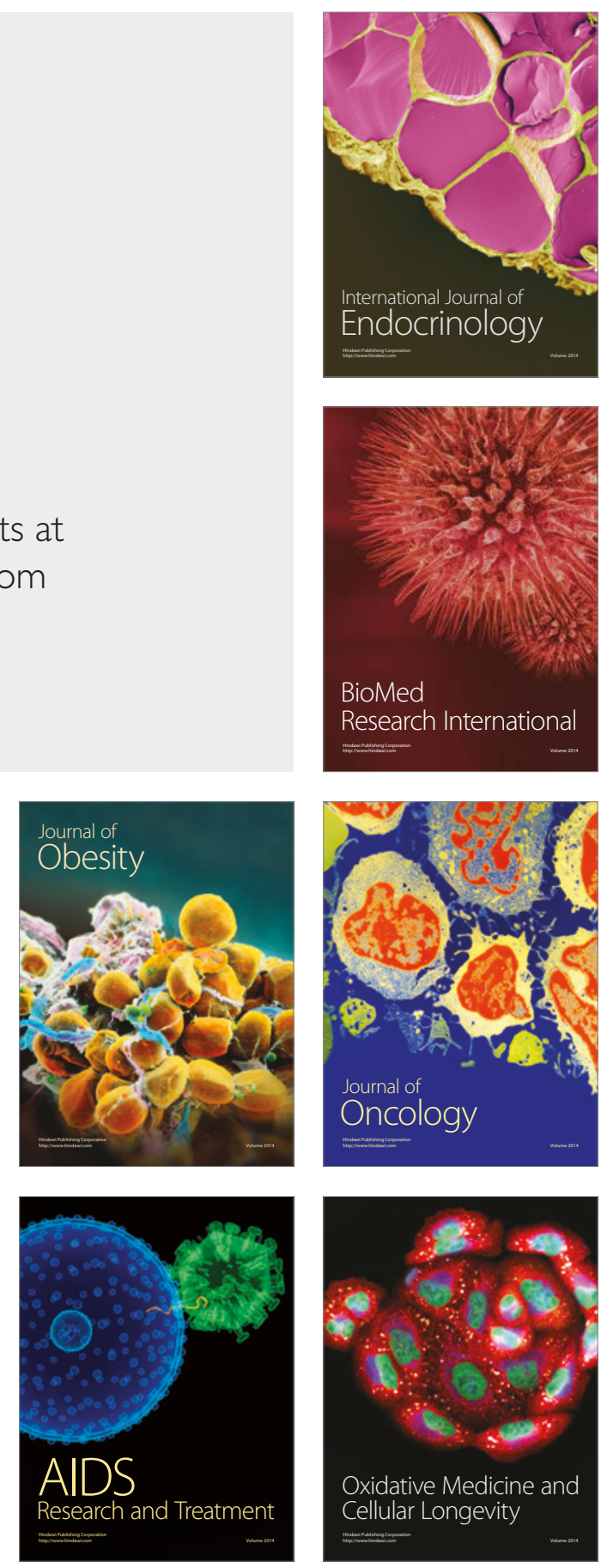